\title{
Oatmeal Powder
}

National Cancer Institute

\section{Source}

National Cancer Institute. Oatmeal Powder. NCI Thesaurus. Code C29298.

Colloidal oatmeal, whole oats milled to a fine powder, with anti-pruritic activity. Oatmeal powder, especially when added to a warm bath, is used to provide temporary relief of itching and irritation caused by insect bites, eczema, sun burns, reactions to poisonous plants and various other skin rashes and allergies. 